Aus der Medizinischen Klinik der Universität in Breslau. (Direktor: Geh. Med.-Rat Prof Dr. Minkowski.)

\section{Ueber Wasserretention bei den Haferkuren der Diabetiker.}

Von Dr. M. Mirowsky.

Plötzliche größere Gewichtszunahmen um einige oder viele Kilogramm in wenigen Tagen sind bei schweren Diabetikern bekannt, besonders wenn wegen Azidose Haferkuren mit Natrium bicarbonicum gemacht werden. Es handelt sich dabei um Gewichtszunahmen infolge von Wasserretention in den Geweben. Die Haut wird feuchter und gespannter; Flüssigkeitsansammlungen in den serösen Höhlen werden nicht honstatiert, dagegen treten nicht so selten sehr erhebliche ödematöse Schwellungen an den unteren Extremitäten auf. Mit Aussetzen der Haferdiät und Uebergang zu einer Fleisch- oder gemischten Kost schwindet diese Gewichtszunahme unter Anstieg der Diurese selbst dann, wenn Natrium bicarbonicum weiter gegeben wird. Die Ursache dieses ,Haferödems “ [v. Noorden (1)] ist unbekannt. v. Noorden selbst rechnet es zu toxischen Oedemen. Auch Minkowski (2) erwähnt es als häufige Folge der Haferkur.

Es werden ja auch sonst bei Diabetikern mitunter auffällige Schwankungen des Körpergewichts infolge von Störungen des Wasserhaushaltes auch bei kohlehydratarmer Kost unabhängig von dem Stickstoffhaushalt beobachtet [Naunyn (3), Stäubli (4), Falta (5), Gigon (6) u. a.]. Die Wasserretention bei Haferkuren ist aber besonders stark und fast gesetzmäßig. Es liegt daher nahe, hier an besondere Vorgänge zu denken.

Blum (7) glaubt, da es sich um Fälle mit Azidose handelt, daß das Natrium bicarbonicum, das gewöhnlich mit der Haferkur gleichzeitig gereicht wird, die Ursache der Wasserretention ist. Es soll das Natrium bicarbonicum zur Neutralisation der Säuren lebhaft von den Geweben an sich gerissen und damit große Mengen Wassers zuriickgehalten werden. Es wäre also nach Ansicht Blu ms das Natrium bicarbonicum die eigentliche Ursache der Oedembildung bei Haferkuren.

Um dieser ungeklärten Frage näher zu kommen, haben wir folgende Untersuchungen angestellt: Wir haben 1. bei Diabetikern verfolgt, ob die Verabreichung von Natrium bicarbonicum oder die Haferkur zur Wasserretention führt, und 2. das Verhalten von nicht diabetischen Individuen, deren Herz und Nieren gesund waren, in ihrem Verhalten bei Haferkuren und Verabreichung von Natrium bicarbonicum bei verschiedenen Diätformen geprüft.

Es wurde bei diesen Versuchen .das Körpergewicht täglich früh nüchtern, dic 24stündige Harnmenge bestimmt. Die Versuchsreihe nicht diabetischer Patienten gestaltete sich folgendermaßen: Patient bekam zwei Tage lang seine gewöhnliche Kost (erste Periode). Dann drei Tage hindurch $250 \mathrm{~g}$ Hafermehl und $50 \mathrm{~g}$ Butter auf fünf Portionen verteilt (zweite Periode). Es folgten (dritte Periode) zwei Tage mit gemischter Kost, wie in der ersten Periode. In der vierten Periode wurden dazu $30 \mathrm{~g}$ Natrium bicarbonicum auf den ganzen Tag verteilt gegeben. In den folgenden drei Tagen (fünfte Periode) erhielten die Patienten $250 \mathrm{~g}$ Hafer $+50 \mathrm{~g}$ Butter und $30 \mathrm{~g}$ Natrium bicar. bonicum. Die sechste Periode entsprach völlig der vierten Periode und dauerte zwei Tage, und in der siebenten Periode wurde wieder zur Anfangskost zurückgekehrt.

Die Flïssigkeitszufuhr war an den Tagen mit Natrium bicarbonicum stets etwas größer als an den anderen Tagen.
Tabelle 1 .

\begin{tabular}{|c|c|c|c|c|}
\hline $\begin{array}{l}\text { Patient Sch. } \\
\text { tagl. Natr. bicar- } \\
\text { bonicum } 50 \mathrm{~g}\end{array}$ & $\begin{array}{l}\text { Patient } \mathrm{Fr} \\
\text { tagl. Natr. bicar- } \\
\text { bonicum } 40 \mathrm{~g}\end{array}$ & $\begin{array}{c}\text { Patient Br. } \\
\text { tagt. Natr. bicar- } \\
\text { bonicum } 50 \mathrm{~g}\end{array}$ & $\begin{array}{l}\text { Patient P. } \\
\text { tagl. Natr. bicar } \\
\text { bonicum } 40 \mathrm{~g}\end{array}$ & \\
\hline $\begin{array}{l}\text { 1. Tag } \\
\text { Gew. } 41,4 \\
\text { Urin } 6260\end{array}$ & $\begin{array}{l}\text { 1. Tag } \\
\text { Gew. } 48,3 \\
\text { Urin } 3300\end{array}$ & $\begin{array}{l}\text { 1. Tag } \\
\text { Gew. } 50,0 \\
\text { Urin } 2900\end{array}$ & $\begin{array}{l}\text { 1. Tag } \\
\text { Gew. } 59,6 \\
\text { Urin } 3300\end{array}$ & \multirow{2}{*}{ strenge Diät } \\
\hline $\begin{array}{l}\text { 2. Tag } \\
\text { Gew. } 41,7 \\
\text { Urin } 5157\end{array}$ & $\begin{array}{l}\text { 2. Tag } \\
\text { Gew. } 48,5 \\
\text { Urin } 3700\end{array}$ & $\begin{array}{c}\text { 2. Tag } \\
\text { Gew. } 50,5 \\
\text { Urin } 2600\end{array}$ & $\begin{array}{l}\text { 2. Tag } \\
\text { Gew. } 60,0 \\
\text { Urin } 2400\end{array}$ & \\
\hline $\begin{array}{l}\text { 3. Tag } \\
\text { Gew. } \\
\text { Urin }\end{array}$ & $\begin{array}{l}\text { 3. Tag } \\
\text { Gew. } 48,0 \\
\text { Urin } 3600\end{array}$ & $\begin{array}{l}\text { 3. Tag } \\
\text { Gew. } 51,5 \\
\text { Urin } 1600\end{array}$ & $\begin{array}{l}\text { 3. Tag } \\
\text { Gew. } 60,0 \\
\text { Urin } 1500\end{array}$ & \multirow{4}{*}{ Hafertage } \\
\hline $\begin{array}{l}\text { 4. Tag } \\
\text { Gew. }\end{array}$ & $\begin{array}{l}\text { 4. Tag } \\
\text { Gew. } 49,0 \\
\text { Urin } 2800\end{array}$ & $\begin{array}{l}\text { 4. Tag } \\
\text { Gew. } 52,8 \\
\text { Urin } 1750\end{array}$ & $\begin{array}{l}\text { 4. Tag } \\
\text { Gew. } 61,6 \\
\text { Urin } 1600\end{array}$ & \\
\hline $\begin{array}{l}\text { 5. Tag } \\
\text { Gew. } 46.1 \\
\text { Urin } 3250\end{array}$ & $\begin{array}{l}\text { 5. Tag } \\
\text { Gew. } 51,2 \\
\text { Urin } 2900\end{array}$ & $\begin{array}{l}\text { 5. Tag } \\
\text { Gew. } 54,5 \\
\text { Urin } 2100\end{array}$ & $\begin{array}{l}\text { 5. Tag } \\
\text { Gew. } 64,0 \\
\text { Urin } 3000\end{array}$ & \\
\hline $\begin{array}{l}\text { 6. Tag } \\
\text { Gew. } 45,7 \\
\text { Urin } 4500\end{array}$ & $\begin{array}{l}\text { 6. Tag } \\
\text { Gew. } 51,5\end{array}$ & $\begin{array}{l}\text { 6. Tag } \\
\text { Gew. } 53,5\end{array}$ & $\begin{array}{l}\text { 6. Tag } \\
\text { Gew. } 64,5\end{array}$ & \\
\hline $\begin{array}{l}\text { 7. Tag } \\
\text { Gew. } 44,4 \\
\text { Urin } 6200\end{array}$ & $\begin{array}{l}\text { 7. Tag } \\
\text { Gew. } 51,2 \\
\text { Urin } 4700\end{array}$ & $\begin{array}{c}\text { 7. Tag } \\
\text { Gew. } 51.9 \\
\text { Urin } 2100\end{array}$ & $\begin{array}{l}\text { 7. Tag } \\
\text { Gew. } 61,2 \\
\text { Urin } 4200\end{array}$ & \multirow{3}{*}{ strenge Diăt } \\
\hline $\begin{array}{c}\text { 8. Tag } \\
\text { Gew. } 41,9 \\
\text { Urin 5310 }\end{array}$ & $\begin{array}{l}\text { 8. Tag } \\
\text { Gew. } 50,0 \\
\text { Urin } 3400\end{array}$ & $\begin{array}{l}\text { 8. Tag } \\
\text { Gew. } 50,7 \\
\text { Urin } 2300\end{array}$ & $\begin{array}{l}\text { 8. Tag } \\
\text { Gew. } 58,0 \\
\text { Urin } 4000\end{array}$ & \\
\hline $\begin{array}{l}\text { 9. Tag } \\
\text { Gew. } 40.5 \\
\text { Urin } 3870\end{array}$ & & & & \\
\hline
\end{tabular}

Aus unseren zahlreichen Beobachtungen an Diabetikern, bei denen es durch Haferkur zu Wasserretention kam, sind in der Tabelle 1 einige Beispiele herausgehoben. Bei diesen Fällen zeigt sich zur Zeit der strengen Diät trotz erheblicher Natrium bicarbonicum-Zufuhr keine Wasserretention, wie der Vergleich mit dem Gewicht bei der Aufnahme und vor der Natrium bicarbonicum-Zulage ergibt. In den Hafertagen steigt sofort das Gewicht an, und die Urinmenge sinkt in den meisten Fällen erheblich. Die Gewichtszunahme kann in 1-2 Tagen $4,5 \mathrm{~kg}$ (z. B. Fall 4) und mehr betragen. Nach Schluß der Haferkur sinkt trotz weiterer Verabreichung von Natrium bicarbonicum bei strenger Diät das Gewicht ebenso schnell unter erheblichem Steigen der Urinmenge.

Aus diesen Tatsachen geht hervor, daß auch bei Diabetischen das Natrium bicarbonicum an sich nicht $z u$ Wasserretention führt. Die Wasserretention ist vielmehr streng an die Hafermehlzufuhr gebunden. Nach Beobachtungen von Blum (10) und persönlichen Mitteilungen von Severin erfolgt auch bei Weizenmehlzufuhr dieselbe Gewichtsvermehrung.

Die Untersuchung der nicht diabetischen Individuen ergab nun folgenden Befund (siehe Tabelle S. 460).

Vergleichen wir die Periode I und II, so sehen wir, daß bei Haferkur keine Gewichtszunahme durch Wasserretention stattfindet. Die Urinmenge nimmt in fünf Fällen dementsprechend zu, in drei Fällen bleibt sie annähernd unverändert. Die Aziditätsverhältnisse des Harns schienen ohne Bedeutung zu sein. Bei Uebergang von Haferzufuhr zu gemischter (fester) Kost (Vergleich der Periode II und III) sinkt umgekehrt in den meisten Fällen die Urinmenge sehr crheblich. In einzelnen Fällen ist die Urinmenge am ersten Tag der Nachperiode crhöht, wahrscheinlich infolge etwas verlangsamter Wasserausscheidung. Das Körpergewicht steigt meist wieder an. Es scheint eher fast in dieser Nachperiode eine gewisse Wasserretention stattzufinden.

Der Vergleich der dritten und vierten Periode ergibt, daß in Zeiten der Natriumzılage die Urinmengen zunehmen, in einzelnen Fällen sehr erheblich (z. B. 7 und 8), wohl zum Teil infolge vermehrter Flüssigkeitszufuhr bei Darreichung von Natrium bicarbonicum. Das Gewicht steigt aber trotzdem in allen Fällen an, mitunter allerdings nur sehr wenig oder nur vorübergahend, am ersten Tage der Natronzulage, um noch während dieser wieder herunterzugehen. Die geringsten Gewichtszunahmen oder sogar Gewichtsverminderungen erfolgen dann, wenn die Diurese sehr groß war. Eine vorübergehendc geringe Wasserretention scheint demnach während der Natronzulage bei gemischter Nahrung vorkommen zu können.

Betrachtung der Periode VI und VII, wo umgekehrt die Natronzulage bei fester Kost ansgesetzt wird, ergibt, daß auch in der sechsten Periode (Natronzufuhr) von einer stärkeren Wasserretention nicht die Rede gewesen sein kann, da die meisten Patienten in der Nachperiode VI dasselbe Gewicht behalten oder sogar etwas an Gewicht zunehmen Auch die Wasserausscheidung bleibt in den meisten Fällen annähernd unverändert. Fine mitunter beobachtete geringe Abnahme ist Folge verminderter Zufuhr. 
T a b e 11 e 2 .

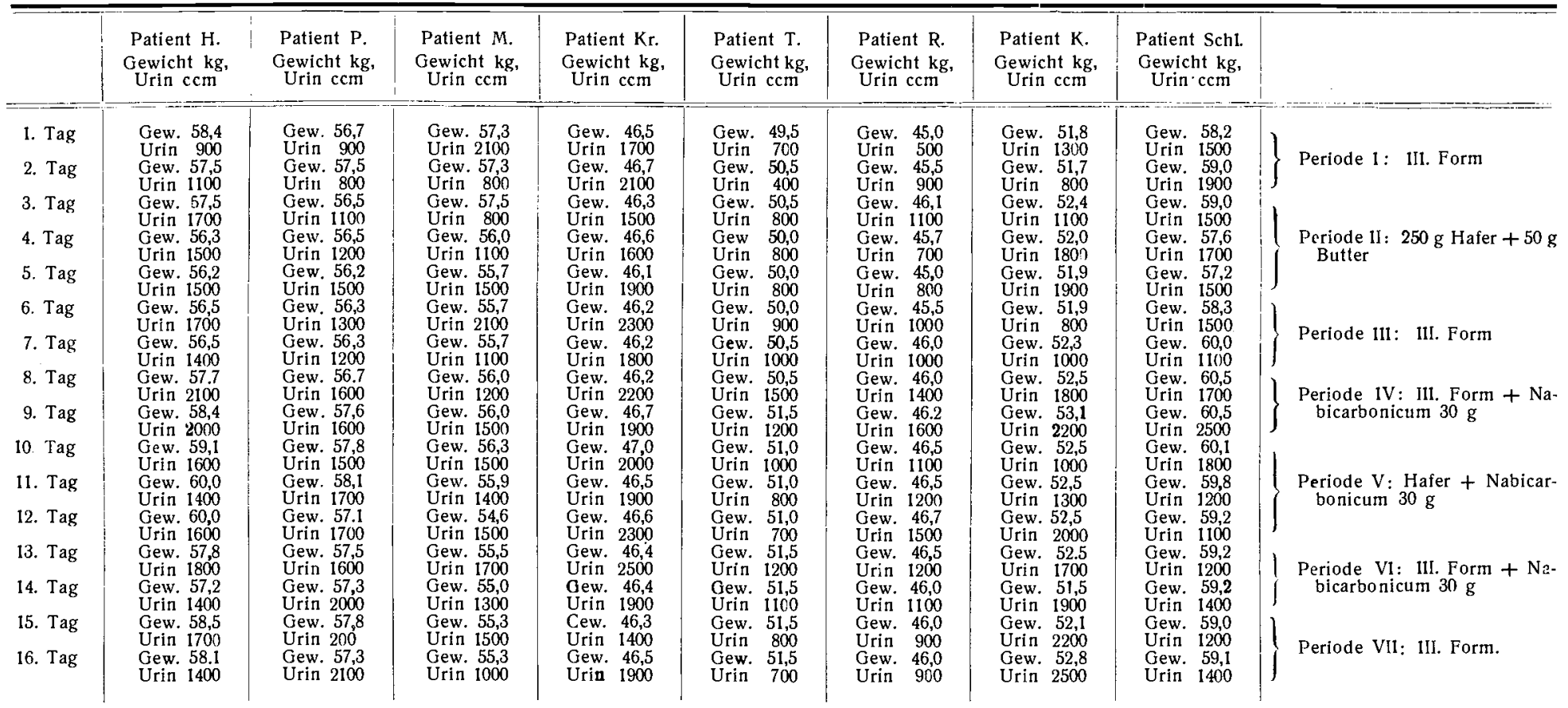

Der Vergleich zwischen Haferkur ohne und mit Natrium bicarbonicum (Periode II und V) ergibt im allgemeinen in der fünften größere Harnmengen als in der zweiten Periode. Auch die Gewichte zeigen im Verlauf der fünften Periode gleichmäßig eine sinkende Tendenz. In zwei Fällen erfolgt eine am ersten Tage der Natronzulage zur Haferkur schon vorübergehende Gewichtszunahme. Hier ist also die Zulage von Natrium bicarbonicum zur Haferkur ohne Einfluß auf die Wasserausscheidung.

Es zeigt sich also bei diesen Versuchen, daß die einfache Haferkur ohne Einfluß auf den Wasserhaushalt des Körpers ist. Auch eine Haferkur mit $\mathrm{Zulage}$ von täglich $30 \mathrm{~g}$ Natrium bicarbonicum führt nicht zur Wasserretention. Zulage von Natrium bicarbonicum bei einer gemischten Diät kann allerdings wohl mitunter zu einer geringen vorübergehenden Wasserretention führen. Diese ist aber jedenfalls sehr gering und nicht gesetzmäßig. Eine merkliche Abnahme der Harnmenge war nicht zu konstatieren, mitunter eher eine auffällige Verniehrung derselben. Diese Beobachtungen stehen völlig im Einklang mit den Versuchen von Blum, der ebenfalls bei gemischter Kost bei normalen Individuen keine Abnahme der Harnmenge bei Natronzulage sah. Nur bei Arteriosklerose wirkte Natronzulage stark wasserretinierend.

Nach längere $Z$ it hindurch verabreichter Milchnahrung konnte Blum (7) durch Zalage von Natrium bicarbonicum eine Wasserretention auch bei Normalen erzielen. Diese Beobachtung wurde von ihm auf eine Salzverarmung des Körpers bezogen. Diesem Befunde widersprechen bis zu einem gewissen Grade unsere Beobachtungen bei den salzarmen Hafertagen, doch könnte vielleicht die verschiedene Versuchungsanordnung diese Differenz erklären. Eine wesentliche oder sicher diuretische Wirkung des Natrium bicarbonicum, wie sie Bergell und Fleischmann (8) beobachteten, sahen wir allerdings, anscheinend auch Bl u $\mathrm{m}(7)$, nicht, nur einige Male (7 und 8) waren die Urinmengen etwas erheblicher. Die gegenteiligen Beobachtungen Pfeifers (9) - erhebliche Wasserretention bei Natronzufuhr - - sind vielleicht so zu erklären, daß es sich bəi seinem ausgesprochenen Falle um eine 65 jährige Person handelte, bəi der wohl Gefäßveränderungen nicht ausgeschlossen sind, die nach den Beobachtungen Blums (7) zur Wasserretention bei Natronzulage disponieren.

Wie die normalen Individuen verhält sich auch ein Knabe mit Diabetes insipidus (s. Tabelle 3).

Während der Hafertage tritt mit und ohne gleichzeitige Verabreichung von Nat-ium bicarbonicum eine Gewichtsabnahme ein (Periode II und IV). Die Harnmenge stieg wohl infolge der vermehrten Flüssigkeitszufuhr: Sie wurde durch die Natronzulage auffallenderweise nicht weiter gesteigert.

Bei gemischter Kost (Periode I, III, V und VI) ist das Gewicht stets höhrr, die Urinmenge kleiner als bei Haferkost, wird aber dureh Natronzulage nicht beeinflußt. Auch hier aber hat das Natron weder wasserretinierende, noch wassertreibende Wirkung (vgl. Periode V und VI).

Es verhält sich also der Diabetiker bei Haferdarreichung entgegengesetzt zum normalen Individuum und zum Kranken mit Diabetes insipidus.

\begin{tabular}{|c|c|c|c|c|c|c|}
\hline $\begin{array}{c}\text { Periode I } \\
\text { Gemischte } \\
\text { Kost }\end{array}$ & $\begin{array}{c}\text { Periode I1 } \\
\text { Hafertage }\end{array}$ & $\underset{\text { Kost }}{\text { Periode III }}$ & $\mid \begin{array}{c}\text { Periode IV } \\
\text { Hafer }+30 \mathrm{~g} \\
\text { Natron } \\
\text { bicarbon. }\end{array}$ & $\begin{array}{c}\text { Periode V } \\
\text { Gemischte } \\
\text { Kost }\end{array}$ & $\mid \begin{array}{c}\text { Periode VI } \\
\text { Gemischte } \\
\text { Kost }+30 \mathrm{~g} \\
\text { Natron } \\
\text { bicarb. }\end{array}$ & $\begin{array}{c}\text { Periode VII } \\
\text { Gemischte } \\
\text { Kost }\end{array}$ \\
\hline \multirow[t]{3}{*}{$\begin{array}{l}\text { 1. Tag } \\
\text { Gew. } 29,0 \\
\text { Urin } 3300^{*} \text { ) } \\
\text { Spez. G. } 1003\end{array}$} & $\begin{array}{l}\text { 2. Tag } \\
\text { Gew. 28, } \\
\text { Urin 5400 } \\
\text { Spez. G. } 1003\end{array}$ & \begin{tabular}{|c|} 
5. Tag \\
Gew. 29,0 \\
Urin 5000 \\
Spez.G. 1003
\end{tabular} & \begin{tabular}{|c|} 
8. Tag \\
Gew. 29,6 \\
Urin 5000 \\
Spez.G. 1005
\end{tabular} & \begin{tabular}{|} 
11. Tag \\
Gew. 28,8 \\
Urin 3700 \\
Spez.G. 1004
\end{tabular} & \begin{tabular}{|} 
13. Tag \\
Gew. 29,2 \\
Urin 4200 \\
Spez. G. 1003
\end{tabular} & $\begin{array}{l}\text { 16. Tag } \\
\text { Gew. } 29,5 \\
\text { Urin } 4500 \\
\text { Spez. G. } 1002\end{array}$ \\
\hline & $\begin{array}{l}\text { 3. Tag } \\
\text { Gew. } 28,8 \\
\text { Urin } 4600 \\
\text { Spez. G. } 1004\end{array}$ & \begin{tabular}{|l|} 
6. Tag \\
Gew. 29,2 \\
Urin 3600 \\
Spez. G. 1003
\end{tabular} & \begin{tabular}{|c|} 
9. Tag \\
Gew. 28,7 \\
Urin 4200 ? \\
3 Spez. G. 1004
\end{tabular} & 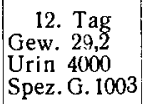 & \begin{tabular}{|c|} 
14. Tag \\
Gew. 29,2 \\
Urin 340')? \\
Spez.G. 1003
\end{tabular} & $\begin{array}{l}\text { 17. Tag } \\
\text { Gew. } 29,6 \\
\text { Urin } 4800 \\
\text { Spez. G. } 1003\end{array}$ \\
\hline & $\begin{array}{l}\text { 4. Tag } \\
\text { Gew. 28.9 } \\
\text { Urin } 5400 \\
\text { Spez.G. } 1004\end{array}$ & \begin{tabular}{|c|} 
7. Tag \\
Gew. 29,6 \\
Urin $4400 ?$ \\
Spez. G. 1004
\end{tabular} & \begin{tabular}{|c|} 
10. Tag \\
Gew 28,5 \\
Urin 3700 \\
4 Spez.G. 1004
\end{tabular} & & $\begin{array}{l}\text { 15. Tag } \\
\text { Gew. } 29,3 \\
\text { Urin } 5900 \\
\text { Spez. G. } 1002\end{array}$ & \\
\hline
\end{tabular}

*) Urinmenge nicht genau festgesteltt, da Pat. nachts das Bett genäbt hat.

Es muß hier also eine bestimmte Veränderung der Gewebe oder der Gefäße bei der Zuckerharnruhr vorliegen, die den Kranken zur Retention von Wasser disponiert.

Daß es sich nicht einfach um ein Heranziehen von Natrium bicarbonicum an die Gewebe mit sekundärer Retention von Wasser handelt, wie $\mathrm{Blum}$ (7) annimmt, geht daraus hervor, daß 1. Natrium bicarbonicum an sich beim Diabetiker mit gemischter Kost nicht zu Wasserretention führt und 2. daß bei vielen dieser Fälle von einer Alkaliverarmung der Gewebe nicht die Rede sein kann, da schon lange vor der Haferkur reichlich Natrium bicarbonicum gegeben wurde und der Harn nicht abnorm sauer war.

$\mathrm{Zu}$ dieser Gewebs- oder Gefäßdisposition des Diabetikers $\mathrm{zu}$ Wasserretention muß aber noch ein zweites Moment hinzutreten, das im Hafermehl (resp. Weizenmehl) zu suchen ist. Vermehrte Flüssigkeitszufuhr allein kann es nicht sein, da reichliches Trinken von Wasser beim Diabetiker nicht zu derartigen Retentionen zu führen pflegt. Wahrscheinlich bestehen engere Beziehungen zwischen Kohlehydraten (Hafer, Weizen, Traubenzucker) und Wasserretention. Darauf weisen auch die Beobachtungen an kleinen Kindern hin, wo wir ebenfalls bei kohlehydratreicher Nahrung Wasserretention beobachten. (Literatur vgl. Meyer (11), Bittorf (12).

Weitere Untersuchungen sollen noch angestellt werden, um das eigenartige Verhalten der Diabetiker näher zu erforschen.

Literatur: 1. v. Noorden, Die Zuckerkrankheit und ihre Behandlung. 2. Minkowski, Verhandlurgen des Kongresses für innere Medizin 1911, Diskussions bericht. - 3. Na uny $n$, Nothnageis Handbuch. - 4. Stäubli, Deutsches Archiv für klinische Medizin, Bd. 93. - 5. Falta und Gigon, Zeitschrilt für klinisclie Medizin 1907, Bd. 61. - 6. Gigon, lbidem, Bd. 63. - 7. Blu m, Verhandlungen des Kongresses für innere Medizin 1909. - 8. Bergell und Fleischmann, Verhandlungen des Kongresses für innere Medizin 1907. - 9. Pfeiffer, Verhandlungen des Kongresses für innere Medizin 1911. - 10. B lum, Manchener medizinische Wochenschrif 1911, No 27. - 11. Meyer, Ergebnisse der inneren
Bd. 1. - 12. Bittorf, Albus Sammlung, Bd. 3, H. 6 . 